\title{
Long-term follow-up of central serous chorioretinopathy
}

\author{
C. MitCHELl Gilbert, SARAH L. OWENS, PATRICIA D. SMITH, \\ AND STUART L. FINE
}

\author{
From Retinal Vascular Center, the Wilmer Ophthalmological Institute, the Johns Hopkins Medical \\ Institutions, USA
}

SUMmaRY The Wilmer Retinal Vascular Center's experience with central serous chorioretinopathy from 1970 to the end of 1979 was reviewed and compared with previous studies. Retrospective analysis of 73 patients seen at follow-up suggests no clinically significant effect of focal argon laser photocoagulation on final visual acuity or recurrence rate. Patients with initial visual acuity of 20/20 remained at that level, and patients with initial visual acuity of less than $20 / 30$ gained, on average, two to three Snellen lines at follow-up. Approximately one-third of both untreated and treated patients had recurrence or presumed persistence during the follow-up period. With the inclusion of episodes that occurred before the first Wilmer Institute visit about half of each group had recurrence or presumed persistence. Recurrences were most often due to leakage from a site within one disc diameter of the original site of leakage.

Central serous chorioretinopathy (CSCR) is an idiopathic serous detachment of the macula caused by focal leakage of choroidal interstitial fluid through the retinal pigment epithelium. Since von Graefe first described central serous chorioretinopathy in $1866^{\prime}$ dozens of papers have reported its demographic characteristics, presenting visual symptoms, and ophthalmological signs. Patients usually report the sudden onset of central blurring, metamorphopsia, and relative scotoma. Serous detachment usually lasts about three months. Focal photocoagulation of the leak site significantly decreases the duration of detachment to about one month, but has no significant effect on visual acuity..$^{2-5}$ Indirect photocoagulation, which has been employed to avoid direct coagulation of subretinal foveal leaks, has no effect on the duration of detachment. ${ }^{56}$

We found in the literature 12 series that included CSCR patients with follow-up of one or more years. ${ }^{2-57-13}$ Seven are retrospective follow-up studies, four are prospective randomised studies, and one is a prospective, nonrandomised study. The studies by Klein et al. ${ }^{10}$ Dellaporta, ${ }^{11}$ and Nanjiani ${ }^{12}$ are most comparable with our study.

Correspondence to Stuart L. Fine, MD, Maumenee 229, Wilmer Ophthalmological Institute, Johns Hopkins Medical Institutions, 600 North Wolfe Street, Baltimore, Maryland 21205, USA.
In our retrospective long-term follow-up study of CSCR patients we studied visual acuity outcome, recurrence tendency, and pigment epithelial leakage pattern and mottling features. We searched for possible prognostic indicators and attempted to determine the long-term effects of focal argon laser photocoagulation.

\section{Patients and methods}

We reviewed 157 cases of CSCR with classic focal retinal pigment epithelial dye leakage and macular detachment that were identified from the Wilmer Retinal Vascular Center files. Cases with coexisting ocular disease were not included. Of these 157 cases of active central serous retinopathy $105(67 \%)$ were not treated and $52(33 \%)$ were treated with focal argon laser photocoagulation. We performed a follow-up examination on approximately half of each group: 47 untreated, 26 treated. We obtained followup for an additional 45 patients from their local ophthalmologists or by detailed questionnaire.

The initial and interim examinations were performed by the Wilmer Retinal Vascular Center staff. All initial examinations included visual acuity measurement (existing correction plus pinhole) by a technician, direct and indirect ophthalmoscopy, contact lens biomicroscopy, and stereoscopic colour 
fundus photography. All but two patients with active CSCR had fundus fluorescein angiography. In patients who were treated, the coherent radiation argon laser was used to produce a light to medium intensity coagulation. Typical treatment parameters were $100-200 \mu \mathrm{m}$ size, 0.1 to $0.2 \mathrm{~s}$ duration, and $100-300 \mathrm{~mW}$ power. At the follow-up examination the same diagnostic procedures were performed by one or two of us (M.G., S.L.F.). Visual acuity was again measured by a technician who did not know whether the patient had been treated or not. Stereo colour photographs were obtained on all patients who returned for follow-up, and fluorescein angiography was obtained on all but four patients. Separately and then together two of us (M.G., S.L.F.) evaluated all colour photographs and fluorescein angiograms for the following: location and size of detachment; number, location, type, and size of retinal pigment epithelial dye leakage; and location, extent, and severity of retinal pigment epithelial mottling. Detachment and leakage characteristics were easily agreed upon. Evaluation of retinal pigment epithelium mottling was much more subjective and therefore will not be discussed.

\section{Results}

Demographic data. The 47 untreated and 26 treated patients seen for follow-up were representative for all baseline data of the 157 patients whose charts we reviewed with respect to the following: percentage treated, age, sex, laterality, onset to first visit, duration, onset to treatment, and potential follow-up duration. We will discuss in detail only the data for patients seen in follow-up.

Basic demographic characteristics were typical of the previously reported series. Median age at initial visit was 43 years, with a range of 27 to 66 years. Men comprised $81 \%$ of the patients. $14 \%$ of the patients had bilateral detachments (not necessarily simultaneously), but in all of these patients one eye was significantly more symptomatic.

Temporal data. Most patients were referred to the Wilmer Institute early in the course of the disorder. For the 47 untreated patients the median delay between onset of symptoms and first visit was three weeks; for the 26 treated patients the median delay was four weeks. Treatment was performed at a median of five weeks after onset of symptoms. Two patients were treated after more than three years of persistent symptoms.

Median follow-up time for the 47 untreated patients was 58 months, with a range of 1 to 10 years. Median follow-up time for the 26 treated patients was 84 months, with a range of 1 to 9 years. The longer median follow-up time for treated patients probably reflected more liberal use of laser photocoagulation for CSCR before the 1974 study by Watzke and Burton, which showed no beneficial effect of treatment on final visual acuity. ${ }^{4}$

Visual acuity. Thirty-five of the 47 untreated patients had 20/20 visual acuity at follow-up, and 19 of the 26 treated patients attained 20/20 visual acuity. For both treated and untreated patients, if the initial visual acuity was greater than or equal to $20 / 30$, there was no net change in visual acuity. Similarly, if the initial vision was less than $20 / 30$, there was an average improvement of between 2 and 3 Snellen lines.

Recurrences. By history, $13(28 \%)$ of the 47 untreated and five $(19 \%)$ of the 26 treated patients had one or more episodes of symptoms consistent with CSCR before the presenting episode. For the other 34 untreated and 21 treated patients the presenting episode was their first symptom. One or more documented recurrences occurred during the follow-up time in $10(21 \%)$ untreated and $9(35 \%)$ treated patients (Table 1). Seven (15\%) of the untreated patients had detachment at the last routine visit and at the follow-up visit, and persistent symptoms during the interim. We presume that these were persistent detachments. Similarly, three $(12 \%)$ of the treated patients had presumed persistent detachment. In 30 $(64 \%)$ of the untreated and $14(53 \%)$ of the treated patients the presenting episode resolved within one year, and there were no subsequent recurrences. Considering both the historical episodes and the documented recurrences, we found that about half of both the untreated $(49 \%)$ and the treated $(54 \%)$ patients had recurrent or presumed persistent central serous chorioretinopathy.

The recurrence frequency of the 45 patients for whom follow-up information was obtained by the local ophthalmologist or by questionnaire agreed closely with the results obtained on the follow-up patients. Of the 30 untreated patients nine $(30 \%)$ had recurrences during the follow-up period. Of the 15 treated patients five $(33 \%)$ had recurrences. Thus we have clinical or historical follow-up information on $73 \%$ of the 105 untreated patients and $79 \%$ of the 52 treated patients. About one-third of both untreated and treated patients had a recurrence or presumed

Table 1 Documented recurrences of central serous chorioretinopathy

\begin{tabular}{lcc}
\hline \multirow{2}{*}{ Number of recurrences } & \multicolumn{2}{l}{ Number of patients } \\
\cline { 2 - 3 } & Untreated & Treated \\
\hline 0 & $30(64 \%)$ & $14(53 \%)$ \\
$\geqslant 1$ & $10(21 \%)$ & $9(35 \%)$ \\
Presumed persistence $\geqslant 1$ year & $7(15 \%)$ & $3(12 \%)$ \\
Median follow-up time (months) & 56 & 78 \\
\hline
\end{tabular}


Table 2 Number of leakage sites in long-term follow-up of central serous chorioretinopathy

\begin{tabular}{|c|c|c|c|c|c|c|}
\hline & & \multirow{2}{*}{$\begin{array}{l}\text { Number of } \\
\text { leakage sites }\end{array}$} & \multicolumn{2}{|l|}{ Untreated } & \multicolumn{2}{|l|}{ Treated } \\
\hline & & & Initial visit & Follow-up visit & Initial visit & Follow-up visit \\
\hline A. & $\begin{array}{l}\text { All active cases } \\
\text { Total }\end{array}$ & $\begin{array}{r}0 \\
1 \\
2 \\
\geqslant 3\end{array}$ & $\begin{array}{l}2 \\
36(80 \%) \\
4 \\
3 \\
45(100 \%)\end{array}$ & $\begin{array}{l}2 \\
29(64 \%) \\
10 \\
4 \\
45(100 \%)\end{array}$ & $\begin{array}{l}0 \\
17(65 \%) \\
5 \\
4 \\
26(100 \%)\end{array}$ & $\begin{array}{l}0 \\
10(38 \%) \\
5 \\
11 \\
26(100 \%)\end{array}$ \\
\hline B. & Recurrent and persistent cases & $\begin{array}{r}0 \\
1 \\
2 \\
\geqslant 3\end{array}$ & $\begin{array}{l}0 \\
20(87 \%) \\
3 \\
0 \\
23(100 \%)\end{array}$ & $\begin{array}{l}0 \\
14(61 \%) \\
8 \\
1 \\
23(100 \%)\end{array}$ & $\begin{array}{l}0 \\
9(60 \%) \\
2 \\
4 \\
15(100 \%)\end{array}$ & $\begin{array}{l}0 \\
3(20 \%) \\
2 \\
10 \\
15(100 \%)\end{array}$ \\
\hline
\end{tabular}

persistence during the follow-up period. When historical episodes before the follow-up time are included, about half of each group had a recurrence or presumed persistence.

Detachment characteristics. The macula was detached at the initial examination in all but two of the patients. Mean detachment size was 2 disc diameters, with a range of 0.75 to 4 disc diameters. The detachments were usually centered about the fovea but often drifted downward with time. The patients occasionally reported an upward-moving relative scotoma. Subretinal yellow precipitates were present at some time during the observation in seven of the 73 patients seen for follow-up. These precipitates were absent at follow-up in all 10 patients with presumed persistent detachment.

Retinal pigment epithelial leakage. The number of retinal pigment epithelial leakage sites, at initial presentation and during follow-up, is summarised in Table 2. Initial fluorescein angiograms available for

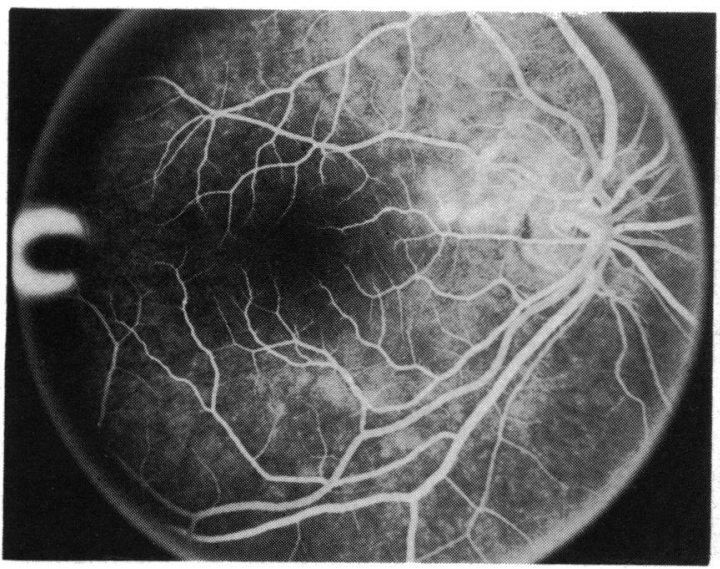

Fig. 1a
45 untreated patients showed no discernible leakage in two patients, one leakage site in 36 patients $(80 \%)$, two leakage sites in four patients, and three or more leakage sites in three patients. During the follow-up period new leakage sites appeared in seven of the patients with only one leakage site initially; $29(64 \%)$ of the untreated patients had only one documented leakage site during the follow-up period.

Of the 23 untreated patients with recurrence or presumed persistence $20(87 \%)$ had only one leakage site initially and three had two leakage sites. Six of the patients initially seen with one leakage site developed other leakage sites during the follow-up period; $14(61 \%)$ of the untreated patients with recurrence or presumed persistence had only one documented leakage site. The untreated patients with recurrence or presumed persistence tended to have similar numbers of leakage sites as those with only one episode which resolved. Treated patients were analysed similarly. Of the 26 treated patients $17(65 \%)$

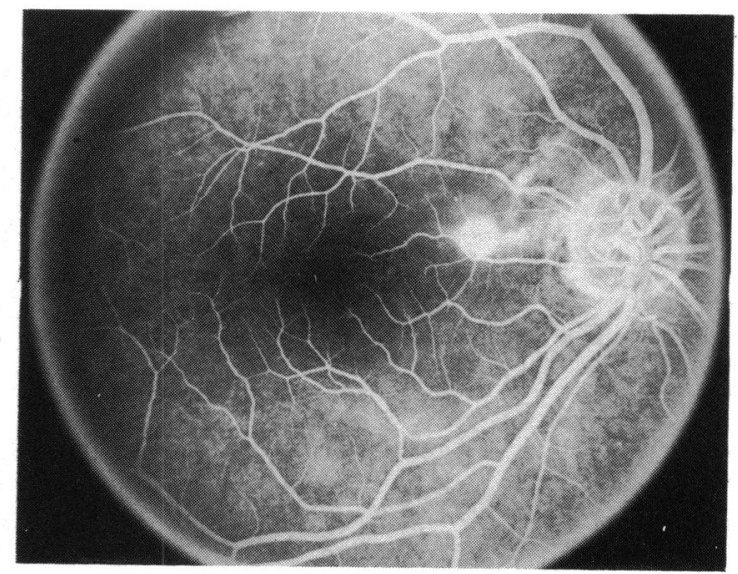

Fig. $1 \mathrm{~b}$

Fig. 1 Fluorescein angiogram showing 'dot' leakage pattern at (a) 26 seconds, (b) 224 seconds. 
had only one leakage site initially, but in the long term $10(38 \%)$ had only one documented leakage site. Treated patients with recurrence or presumed persistence had one initial leakage site in nine $(60 \%)$ cases but in only three $(20 \%)$ cases in the long-term. The patients selected for treatment were more likely to have multiple initial and subsequent leakage sites.

The distribution of pretreatment leakage sites is similar to the previously reported series. ${ }^{10}{ }^{14}$ Of the 45 untreated patients with baseline fluorescein angiograms, leakage sites were distributed as follows: foveal avascular zone, 8; extrafoveal macula (i.e., one disc-diameter centered about the foveal avascular zone), 27; papillomacular bundle, 4 ; elsewhere in the posterior pole, 6 . Leakage sites in treated patients were distributed as follows: extrafoveal macula, 16; papillomacular bundle, 5 ; elsewhere in the posterior pole, 5. No patient with leakage from the foveal avascular zone was treated.

We recognised four distinct types of leakage: a gradually enlarging dot of hyperfluorescence which began from a site smaller than the diameter of a retinal vein (Fig. 1), pinpoint leakage ascending to form a classical 'smokestack plume' (Fig. 2), diffuse retinal pigment epithelial leakage, and pinpoint leakage from a pigment epithelial detachment. At initial presentation the 45 untreated patients with angiograms had the following distribution of leakage types: dot, 33; 'smokestack,' 9; diffuse, 0; dot arising from a pigment epithelial detachment, 3 .

We were particularly interested in the location of the leakage sites in recurrent and presumed persistent detachments relative to the initial leakage sites. Four of the 10 untreated patients with documented recurrent detachment had leakage from the original

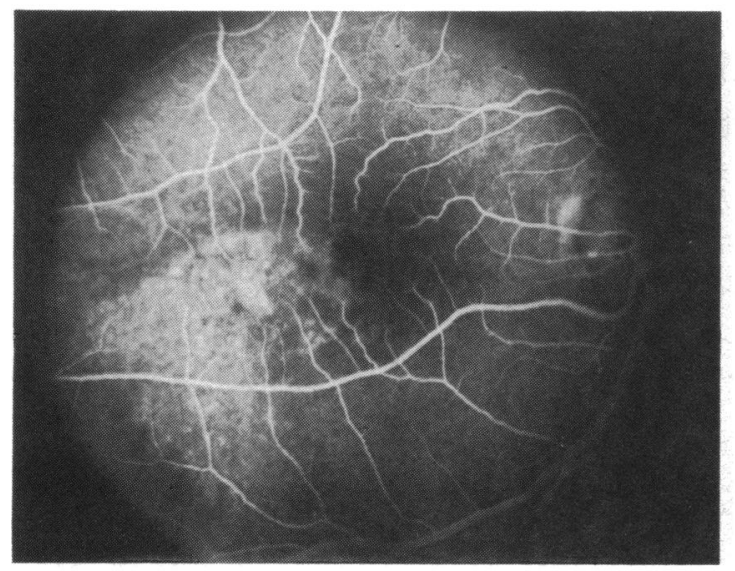

Fig. 2a

Fig. 2 Fluorescein angiogram showing 'smokestack' leakage pattern at (a) 21 seconds, (b) 164 seconds. site, three had new sites within $0 \cdot 5$ disc diameter of the original, and one had a new leakage site at a distance of 2 disc diameters from the original site. In two cases fluorescein angiograms of the recurrent detachment were not available. Of the seven untreated patients with presumed persistent detachment at follow-up five had leakage from the same site and three had leakage from new sites within 0.5 disc diameter of the original site. (One patient had leakage at the original site and from a new site.) Of the 26 treated patients only one had recurrent leakage from a treated site. The adequacy of treatment in this case is questionable owing to the absence of posttreatment scarring. Four treated patients had recurrent leakage from sites within 0.5 disc diameter of the original site, and four had leakage within the next 0.5 disc diameter. In two patients recurrent detachment was caused by leakage at sites which had closed spontaneously without treatment. Presumed persistent detachments in treated patients were associated with diffuse, slow leakage from the treatment site in one patient, new sites within 0.5 disc diameter of the initial sites in two patients, and a new site within the next $0 \cdot 5$ disc disc diameter in one patient. One treated patient with presumed persistent detachment at follow-up had leakage from a site which had previously closed spontaneously without treatment.

Complications. The only complication of laser photocoagulation was the development of relative paracentral scotomas, to which patients adapted well. No case of treated active CSCR seen for follow-up had developed choroidal neovascularisation, although Schatz et al. reported 27 cases with this complication. ${ }^{16}$

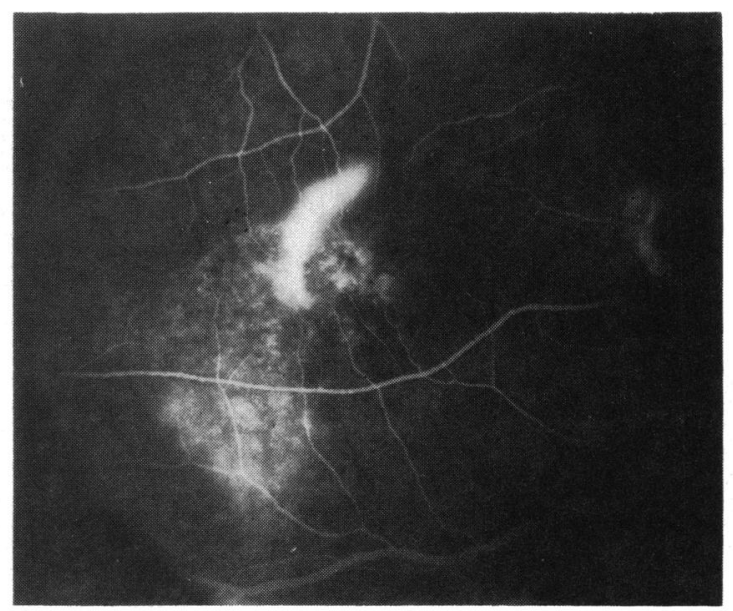

Fig. 2b 


\section{Discussion}

VISUAL ACUITY

This study agrees with all previous reports that the visual prognosis for patients with CSCR, untreated and treated, is favourable. We were impressed by the range of visual acuities with similar-appearing macular detachments. Also, despite 'good' final visual acuities, patients were seldom asymptomatic after resolution of the serous detachment. CSCR is a unique serous macular detachment disorder, following an often recurrent course without serious loss of visual acuity in most cases.

\section{RECURRENCE}

Previous CSCR studies have considered recurrence rates and the effect of laser treatment on these rates. ${ }^{2-57-13}$ It is difficult to compare the papers owing to different or unspecified durations of follow-up and to different criteria for recurrence-i.e., documented and historical. The papers reported recurrence in $25 \%$ to $50 \%$ of untreated patients. In the two most rigorous retrospective long-term studies Dellaporta and Nanjiani documented recurrences in about half of each group of untreated eyes. ${ }^{11} 12$ Fewer of our untreated eyes had recurrence $(21 \%$, all documented), but $15 \%$ had presumed persistent detachment. We cannot explain the lower recurrence rate, especially over a longer follow-up time. By considering previous historical episodes, documented recurrence, and presumed persistent detachment we found that about half $(51 \%)$ of the untreated patients had a single resolving episode of detachment, and about half $(49 \%)$ had a more complicated course.

In their prospective, randomised trial of ruby laser photocoagulation Watzke et al. found no significant effect of treatment on recurrence rate. ${ }^{3}$ The sample size was small, and follow-up time was short (median -23 weeks). Nanjiani found that 'about half' of untreated and treated groups had recurrence. ${ }^{12}$ Dellaporta concluded from his prospective, nonrandomised study of 68 patients that recurrences are 3.3 times more likely in untreated than in treated eyes; therefore he strongly recommended treatment. " Landers ${ }^{13}$ found a recurrence rate of $9 \%$ over a median follow-up of 15 weeks in his study of 33 eyes treated with laser phocoagulation. At the 1980 meeting of the American Academy of Ophthalmology, Spitznas reported a decreased recurrence rate from $32 \%$ to $9 \%$ in untreated versus treated eyes, respectively. Xenon arc photocoagulation, rather than laser photocoagulation, was used. ${ }^{15}$ Most recently Robertson and Ilstrup showed in a prospective randomised study of 42 eyes that untreated and indirectly laser-treated eyes had a recurrence rate of $34 \%$ over 18 months, but no eye treated with focal argon laser photocoagulation had a recurrence. ${ }^{5}$ There are probably two main reasons for the variable recurrence rates: (1) different follow-up durations, and (2) different treatment techniques (especially size of laser spot).

Recognising the weaknesses of retrospective studies we believe that our untreated and treated groups are similar enough to make conclusions about the effect of direct laser photocoagulation on recurrence rate. Both groups had reported a previous episode in about $25 \%$ of cases: untreated $28 \%$, treated $19 \%$. Documented recurrences occurred slightly more often in treated (35\%) than in untreated $(21 \%)$ patients. This difference might be due to the longer median follow-up time available for treated patients. Presumed persistence occurred in about $15 \%$ of both untreated and treated groups. The results of followup by the local ophthalmologist and by questionnaire confirm those of documented follow-ups. Focal argon laser photocoagulation did not appreciably decrease the recurrence rate of CSCR in our non-randomised, though comparable, patient groups. Our findings of recurrent leakage sites explain how this is possible.

The number and relative locations of leakage sites are important factors which are related to the effect of focal laser photocoagulation on recurrence rate. Our treated patients more often had multiple leakage sites at the initial examination than did our untreated patients (35\% versus $16 \%$ respectively). Multiple leakage sites may have been used as a relative indication for treatment. When the entire follow-up period for each group is considered, treated patients still more often had multiple leakage sites than did untreated patients (62\% versus $31 \%$ respectively). When only the treated and untreated patients who had recurrence or presumed persistence $(80 \%$ versus $40 \%$, respectively) are considered, this difference is found to have also existed during the follow-up period.

In the 17 untreated patients with documented recurrence or presumed persistence leakage was occurring at new, adjacent sites about as often as at the original site. This suggests a disorder of the adjacent retinal pigment epithelium which predisposes it to leakage. Leakage occurred at treated sites in only two cases. However, new leakage sites appeared within 1 disc diameter of original treated sites often enough to nullify completely the low recurrence rate from treated sites. New leakage sites always appeared in regions of retinal pigment epithelial mottling, but these sites were otherwise uncharacteristic before leakage appeared. Our study confirms that of Spitznas, ${ }^{15}$ who showed that recurrent leakages arise adjacent to original sites.

Pretreatment fluorescein angiograms of all active cases showed focal or multifocal dye leakage. In 
agreement with previous studies, most leakage (73\%) appeared as slowly enlarging pinpoint dots of hyperfluorescence. Classical 'smokestack' leakage transformed to dot leakage in three cases, but no reverse transformations occurred. 'Smokestack' leakage may indicate a higher leakage rate and lower density of the dye than the subretinal serous fluid. Focal leakage from pigment epithelial detachments was present in many patients. This supports Gass's hypothesis of a common pathology for pigment epithelial detachments and CSCR. ${ }^{17}$ We are impressed, however, that before treatment dye always leaked from a site smaller than typical pigment epithelial detachments, and the pigment epithelial detachments never had diffuse leakage into the subretinal space.

\section{CONCLUSION}

The results of this retrospective follow-up study confirmed the findings of several previous studies concerning epidemiological features, natural course of untreated eyes, prognosis for treated eyes, and absence of difference in final visual acuity between treated and untreated eyes. However, we found no significant difference in recurrence rate between treated and untreated eyes and no significant complications of argon laser photocoagulation. We shall be interested in a follow-up of Robertson and Ilstrup's study group ${ }^{4}$ in five to 10 years.

We are grateful to Maureen Maguire, $\mathrm{PhD}$, for advice regarding data interpretation and presentation, and also to Judy Belt and Richard Belt for colour photography. and fluorescein angiography. Lee Bridgeforth and Lillian Fox assisted in the preparation of the manuscript.

\section{References}

1 von Graefe A. Ueber centrale recidivirende retinitis. Albrecht von Graefes Arch Klin Ophthalmol 1866; 12: 211-5.
2 Leaver P, Williams C. Argon laser photocoagulation in the treatment of central serous retinopathy. Br J Ophthalmol 1979; 63: $674-7$.

3 Burton TC. Central serous retinopathy. In: Blodi FC, ed. Current concepts in ophthalmology. St Louis: Mosby, 1972: 3: 1-28.

4 Watzke RC, Burton TC, Leaverton PE. Ruby laser photocoagulation therapy of central serous retinopathy. Trans Am Acad Ophthalmol Otolaryngol 1974; 78: 205-11.

5 Robertson D, Ilstrup D. Direct, indirect and sham laser photocoagulation in the management of central serous retinopathy. Am J Ophthalmol 1983; 95: 457-66.

6 Watzke RC, Burton TC, Woolson RF. Direct and indirect photocoagulation of central serous retinopathy. Am J Ophthalmol 1979; 88: 914-8.

7 Mitsui Y, Sakanashi R. Central angiospastic retinopathy. Am J Ophthalmol 1956; 41: 105-13. Y

8 Straatsma BR, Allen PA, Pettit TH. Central serous retinopathy. Trans Pac Coast Otoophthalmol Soc 1966; 47: 107-25.

9 Nordholm I. Central serous retinitis. Acta Ophthalmol (Kbh) 1969; 47: 890-9.

10 Klein M, Van Buskirk M, Friedman E, et al. Experience with non-treatment of central serous retinopathy. Arch Ophthalmol 1974; 91 : 247-50.

11 Dellaporta A. Central serous retinopathy. Trans Am Ophthalmol Soc UK 1976; 74: 144-51.

12 Nanjiani M. Long-term follow-up of central serous retinopathy. Trans Ophthalmol Soc UK 1977; 97: 656-61.

13 Landers M. Argon laser treatment of central serous chorioretinopathy. Ann Ophthalmol 1977; 9: 1567-72.

14 Shimizu K, Tobari I. Central serous retinopathy dynamics of subretinal fluid. Mod Probl Ophthalmol 1971; 9: 152-7.

15 Spitznas M. Paper presented at the Annual Meeting of the American Academy of Ophthalmology, Chicago, 1980.

16 Schatz H, Yannuzzi LA, Gitter KA. Subretinal neovascularization following argon laser photocoagulation treatment for central serous retinopathy: complication or misdiagnosis? Trans Am Acad Ophthalmol Otolaryngol 1977; 83: 893-906.

17 Gass JDM. Pathogenesis of disciform detachment of the neuroepithelium. II: Idiopathic central serous retinopathy. Am J Ophthalmol 1967; 63: 587-615. 